\title{
Arthroscopic Multichannel Combined Approach in The Treatment of Knee Lumen Infection With Calf Effusion Abscess $₫ A$ Retrospective Analysis of 15 Patients
}

\author{
Yuan Li \\ Affiliated Hospital of Southwest Medical University \\ Fuyuan Deng \\ Affiliated Hospital of Southwest Medical University \\ Juncai Liu ( $\square$ 1220223982@qq.com ) \\ Affiliated Hospital of Southwest Medical University \\ Peng Zhou \\ Affiliated Hospital of Southwest Medical University \\ Lei Lei \\ Affiliated Hospital of Southwest Medical University \\ Zhong Li \\ Affiliated Hospital of Southwest Medical University
}

\section{Research Article}

Keywords: Knee joint, Infection, Arthroscopy, Abscess

Posted Date: January 22nd, 2021

DOI: https://doi.org/10.21203/rs.3.rs-148437/v1

License: (c) (1) This work is licensed under a Creative Commons Attribution 4.0 International License.

Read Full License 


\section{Abstract}

Background: It is rare to see knee lumen infection with calf effusion abscess. Traditional open surgery is traumatic, slow and difficult to recover. And there are few reports of using arthroscopy to diagnose and treat knee lumen infection with calf effusion abscess. This case series aims to introduce the clinical outcomes of arthroscopic treatment of knee lumen infection with calf effusion abscess.

Methods冈fifteen patients with knee joint cavity infections and calf abscesses from from January 2015 to December 2019, were included(11 males and 4 females; age range from 33 to 61 , the average age is 46.6years). All patients were treated with arthroscopic multi-channel combined approach. The erythrocyte sedimentation rate (ESR) and C-reactive protein (CRP) were recorded before and at the last follow-up. The knee function was evaluated according to the visual analogue scoring method (visual analogue scale, VAS) pain score and Lysholm score.

Results 囚fifteen patients were followed up from 12 months to 2 years, and the average follow-up time was 18.4 months. The operation was smooth in all patients, the incisions were healed at grade I, no postoperative recurrence, no nerve damage, and no complications such as knee stiffness. At the last follow-up, ESR decreased from preoperative $(44.6 \pm 10.1)$ to $(11.4 \pm 3.1)(P<0.001)$, and CRP decreased from preoperative $(30.2 \pm 8.0)$ to $(3.9 \pm 1.0)(P<0.001)$. Lysholm score was reduced by Before $(46.9 \pm 7.7)$ improved to $(93.0 \pm 8.1)(P<0.001)$, the VAS score decreased from $(7.0 \pm 0.9)$ before operation to $(2.1 \pm 1.2)$ $(P<0.001)$, and the differences were statistically significant.

Conclusion囚Arthroscopic multi-channel combined approach is a safe, effective and minimally invasive treatment method for knee joint cavity infection with calf abscess.

\section{Background}

The global annual incidence of infectious arthritis is about $0.006 \%$, of which the knee joint is most commonly affected, and the incidence is on the rise. $78 \%$ of patients live in poverty-stricken areas, and the mortality rate amounts to $11 \%[1,2,3,4]$. Knee joint infection and calf abscess is caused by the increase of pus in the joint and the increase of pressure, and the pus flows down along the muscle space and anatomical fissures. This kind of situation is rare in clinical practice. If the infection persists for a long time, it will cause local bone sclerosis, adhesion of surrounding soft tissues, fibrosis, and eventually damage to the knee joint, stiffness and loss of knee joint function, and the prognosis is extremely poor. Traditional open surgery is traumatic, bedridden for a long time, difficult to recover, easy to stiff joints, and high recurrence rate $[5,6]$. With the increasing maturity of arthroscopic technology, arthroscopic debridement and lavage drainage have shown good short-term effects in the treatment of knee joint infections [7, 8]. Compared with traditional open surgery, arthroscopic surgery has the advantages of minimally invasive, broader and clearer vision, more thorough removal of lesions, easier early recovery and weight bearing $[7,9]$. At present, there are rare reports at home and abroad about the clinical research 
of arthroscopic multi-channel combined approach for the treatment of knee joint cavity infection with calf abscess.

This article is aimed at 15 cases of knee joint cavity infection with calf abscesses admitted to our hospital from January 2015 to December 2019. All of them were treated with arthroscopic multi-channel combined approach surgery and achieved good short-term results. The report is as follows.

\section{Methods}

Inclusion criteria include: The patients were diagnosed preoperatively as having a fluid abscess in the leg caused by knee joint infection, and all patients had no anatomical variation. The symptoms of conservative treatment were not significantly relieved, and there was no obvious surgical contraindication. He was willing to accept arthroscopic surgery and was willing to implement our department's rehabilitation and follow-up plan and signed an informed consent.

Exclusion criteria include: Gout, rheumatoid, hemophilia and other non-infectious knee joint diseases; total knee joint infection with knee joint stiffness; knee joint infection without calf infusion abscess; patients who cannot tolerate surgery; patients with missing data; do not receive rehabilitation from our department Follow the planned patient visit.

From January 2015 to December 2019, a total of 29 patients met the inclusion criteria, 11 males and 4 females, age range from 33 to 61 years, with an average age of 44.1 years. The course of the disease was 4.4 months. There were 10 cases on the left knee and 5 cases on the right knee. On admission for physical examination: knee red, swollen, hot, painful, semi-flexed position, positive floating patellar test, positive joint space tenderness, limited flexion and extension activities. The arthrocentesis fluid was purulent, and WBC, NEUT\%, CRP, and ESR were all elevated. All patients underwent joint cavity puncture, and synovial fluid underwent bacteriological culture and antibiotic sensitivity test. The patient's preoperative knee joint examination is shown in (Table 1).

\section{Surgical purpose and technique}

All operations were performed by the same physician who was proficient in arthroscopic techniques. The patient was under general anesthesia, supine position, routine disinfection of the operation area, sterile towels, postural exhumation, and inflation to $250 \mathrm{mmgH}$. Knee joint cavity cleanup: establish anterior internal and anterior external approaches to the knee joint, perform joint cavity exploration and clean up, take the diseased synovial tissue and send bacteriological smears, tissue culture, and pathological biopsy. Fully clean up the suprapatellar sac, medial and lateral recesses, intercondylar fossa, upper and lower surface of meniscus, cruciate ligament space and other lesions. Under the surveillance of arthroscopy, establish a posterior medial and lateral approach to fully clean the lesion tissue of the posterior internal and external compartments. Debridement of internal calf abscess: Under the surveillance of arthroscopy, open the joint capsule with a planer at the back of the posterior medial joint capsule, and insert the arthroscopy into the abscess cavity under the guidance of the exchange rod to 
establish the first low-inferior approach. Use a planer to clean the tissue of the abscess. According to the downward extension of the abscess and the maximum distance that the planer can clean down, determine how much lower approaches to establish further down, and finally achieve the complete removal of the medial posterior abscess. Debridement of lateral calf abscess: Under arthroscopic monitoring, a high posterolateral approach was established at $0.5 \mathrm{~cm}$ anterior and inferior to the femoral stop of the popliteal tendon. Under arthroscopy, the hole was reamed through the posterolateral approach to the upper edge of the popliteal tendon hiatus. Extend the curved planer to clean up the popliteal tendon hiatus and downflow abscess. After the wound was cleaned, $0.5 \%$ iodophor was soaked for 5 minutes, and then washed repeatedly with normal saline. In the case of Mycobacterium tuberculosis infection, inject $3 \mathrm{mg}$ isoniazid into the joint cavity and indwell one joint cavity and one plasma drainage tube for the pus cavity; in other cases, only indwell the joint cavity plasma drainage tube. The wound is sutured and bandaged with sterile auxiliary materials. Typical cases are shown in (Fig.1). Nutritional support treatment is given after surgery, such as a high-protein diet. Routine postoperative prevention of deep vein thrombosis, analgesia and ice application of lower limbs. According to the results of the drug sensitivity test, sensitive antibiotics were administered intravenously for 2 weeks and then changed to oral administration for 4-6 weeks. ESR and CRP were reviewed every 3 days until the patient's blood indicators were normal. If the local symptoms and signs disappear, the plasma drainage tube can be removed if there is no bacterial growth after the drainage culture. Within 8 hours after surgery, start quadriceps contraction and ankle pump exercises (3 4 times/day, 5-10min/time), and patella push (3 4 times/day, $1 \sim 2 \mathrm{~min} /$ time); On the second day, start non-weight-bearing active and passive knee range of motion training (2 3 times/day, each time reaching the maximum), and at the same time instruct patients with weight-bearing training (1 2 times/day, 5 10min/time). During the hospital stay, daily rehabilitation training was conducted under the guidance of the rehabilitation specialist.

\section{Observation indicators and statistical analysis}

All patients were followed up for more than 12 months, and preoperative and follow-up were scored and recorded by the same physician. The follow-up time was 1, 2, 3, 6, 12, and 24 months after surgery. The ESR value, CRP value, Lysholm score, and VAS pain score were recorded before treatment and at the last follow-up, and analyzed. Data were compiled and analysed using the software SPSS version 20.0 and Microsoft Excel 2010. The enumeration data was expressed with mean \pm standard deviation. Before and after treatment VAS pain score, Lysholm score, ESR value, and CRP value were conducted by paired t test. The level of statistical significance was set at $5 \%(p<0.05)$. The study plan was approved by the Clinical Trial Ethics Committee of the Affiliated Hospital of Southwest Medical University (batch number: KY2020243).

\section{Results}

All the patients in this study were followed up. The follow-up time was 12-24 months, with an average of (18.4 \pm 3.1$)$ months. All the patients had a smooth operation, the incision was healed in stage I. There was no postoperative recurrence, no nerve damage, and no complications such as knee stiffness. At the last 
follow-up evaluation,. The ESR value, CRP value, Lysholm score, and VAS pain score were significantly improved compared to before treatment (Table 2).

\section{Case}

A male, 39 years old, had left knee pain, swelling and limited mobility for $4+$ month. The diagnosis of Septic arthritis of the left knee joint with formation of an infusion abscess in the calf has been considered. The preoperative VAS pain score was 6 points. MRI showed that there was fluid in the articular cavity of the left knee, in which the bursa effusion of the medial head-semimembranosus muscle of the gastrocnemius muscle extended to the subcutaneous inner side of the upper left leg. On July 23, 2018, the patient underwent arthroscopic exploration of the left knee joint cavity + calf injection abscess resection + lesion synovial biopsy. The patient had no obvious postoperative complications and was given sensitive antibiotics intravenously for 2 weeks and then changed to oral administration for 4-6 weeks. After 12 months of follow-up, the symptoms of the knee joint improved significantly (Fig. 1).

\section{Discussion}

The most important finding in this study is that the arthroscopic multi-channel combined approach for the treatment of knee joint cavity infection with calf infusion abscess is a safe and effective surgical method with high overall satisfaction. Knee joint infection is a joint inflammation of the knee caused by microbial infection such as bacteria. The pathogenic factors can be divided into primary and secondary. Primary infection is caused by the invasion of bacteria from other infected sites, blood or lymphatic system. Secondary infections are local puncture or trauma that lead to direct bacterial invasion. Complications of knee joint infection: joint degeneration, soft tissue injury and contracture, osteomyelitis, joint fibrous and bony ankylosis, severe consequences are sepsis and death. Therefore, early diagnosis and treatment are the decisive factors for the final outcome [10]. Knee abscess can extend to the calf and even the foot through the deep and shallow space on the back of the tibia. There are two obvious flow channels from the knee to the calf. One is formed by wrapping the posterior medial path of downward flow from the rupture of the joint capsule. The other is the posterolateral path through the popliteal tendon hiatus.

The main symptoms of knee joint infection with peri-knee abscess are: local pain, swelling, and limited mobility, with an incidence of $78 \%-85 \%$ [11]. Common signs are: joint tenderness, effusion, redness and swelling, and decreased passive mobility. Often in flexion due to pain and effusion. In imaging examinations, knee MRI is the most important examination method for the diagnosis of soft tissue abscesses [12]. The advantages of knee MRI application are: (1) Observe whether the abscess is connected to the joint cavity, which is of reference value for the selection of arthroscopic surgery; (2)The resolution of the soft tissue in the joint is high, the position, size and scope of the abscess can be clearly displayed, and other substances can be identified Sexual mass. Patients in this group were treated with diagnostic antibiotics first, and finally the antibiotic regimen was adjusted according to the results of drug sensitivity to ensure the exact curative effect. The most common bacterial culture result is 
Staphylococcus aureus, followed by Streptococcus and other Gram-positive bacteria $[13,14,15,16]$. As shown in Table 1, 9 of the 15 patients in this study (60\%) had preoperative and postoperative bacterial culture results of Staphylococcus aureus, which was basically consistent with $50 \%$ to $60 \%$ reported in the literature. It is worth noting that 1 patient $(6.6 \%)$ had a bacterial culture result of Staphylococcus epidermidis. Analyzing the reason for its infection of this normal flora may be the weakened immunity of elderly patients. The diagnosis of knee joint infection with calf abscess should be comprehensively evaluated based on the patient's medical history, clinical symptoms, signs, imaging findings and laboratory indicators. In laboratory examinations, dynamic monitoring of changes in ESR and CRP can be used to judge the control of the disease and guide the course of antibiotic use. ESR and CRP at the last follow-up of this study were significantly lower than those before surgery;

Arthroscopic surgery is currently a more recognized surgical method for the treatment of knee joint infections $[7,8,9]$. But for patients with calf abscess, most clinicians will choose traditional open surgery, with stage I drainage of the abscess and stage II resection of the cyst wall tissue. If there are abscesses both inside and outside the knee joint, the traditional open surgery using a single approach cannot completely clean the popliteal tendon hiatus area. It is often necessary to expand the wound and change the position during the operation, which is prone to contamination. Arthroscopic technology can enter through the interstitial space and clean the area smoothly, thereby avoiding damage to important tissue structures. Nair et al [17]. showed that the cure rates of joint puncture/lavage and traditional incision were $79 \%$ and $84 \%$, respectively. All patients in this group did not undergo lavage and drainage after surgery. The reason was that the calf muscle gap was large, and the lavage drainage fluid would penetrate into the muscle gap, which would aggravate the swelling and affect recovery. Faour et al. [7]conducted a study on 695 patients with knee joint infections through the ACSNSQIP database and showed that compared with open irrigation and debridement, patients undergoing arthroscopic surgery had lower blood transfusion rates and adverse events within 30 days after surgery. , The discharge rate is better, and the short-term effect is good. In this study, the arthroscopic multi-channel combined approach was used to treat knee joint cavity infection with calf injection abscess. It has the following advantages: (1)Small incision on the side of the calf, short healing time, small healing scar, has a certain cosmetic effect, and relieves the patient's psychology and economy burden. (2)The internal and external multiple approaches above the calf are converted into the back of the calf to inject the abscess area, which can provide clear and broad intraoperative vision and operation space, fully clean up the lesion tissue in the joint, and more effectively inject the popliteal fossa and the abscess. The regional capsule wall is thoroughly cleaned and flushed. (3)Easy to operate, no need to carefully dissect the rear structure of the calf, which can shorten the operation time. (4)There is no need to continuously change the position, open the patella, and repeatedly disinfect the drape during the operation, which greatly reduces the spread of infection and the risk of recurrence. Therefore, compared with traditional open surgery, the arthroscopic multi-channel combined approach treatment method is more in line with the concept of minimally invasive treatment, cleans up more thoroughly, reduces the occurrence of postoperative adhesions, and reduces deep vein thrombosis, joint stiffness, muscle atrophy and other complications[7,8,18,19]. Johns et al. [16] found that arthroscopic treatment can produce better postoperative range of motion and shorter hospital stay. 
Bohler et al. [20] also showed that the knee mobility of patients undergoing arthroscopic debridement was significantly improved. The postoperative pain of 15 patients in this group was significantly relieved. According to the Lysholm score, the knee function was excellent in 12 cases, good in 2 cases, and fair in 1 case. The excellent and good effective rate was $93.3 \%$. This is basically consistent with the recently reported cure rate of $93-97 \%[7,19]$. However, it is worth noting that postoperative drainage, early rehabilitation, and the use of antibiotics in the foot treatment are essential and important links for the recovery of the disease $[9,14,15]$; it also raises the doctor's proficiency in arthroscopic techniques Higher requirements, such as accidental operation and easy damage to blood vessels, nerves and muscles.

The main points of the operation of the arthroscopic multi-channel combined approach during the operation are: (1)The posterolateral approach should be established slightly higher than usual by about $0.5 \mathrm{~cm}$ in order to obtain a wide enough field of view; (2)Clean the posterior and popliteal tendon hiatus area when necessary Use curved planer heads and control the attractive force of the planer to avoid damaging the front and rear fibers of the meniscus and popliteal tendon hiatus, and avoid damaging the stability of the meniscus; (3)Pay attention to the lower surface of the meniscus and the area between the cruciate ligaments, the cruciate ligament and the femoral condyle When necessary, the area is also cleaned with a curved planer head or even with a $70^{\circ}$ mirror. (4)The medial abscess should be cleaned down as far as possible in accordance with the arthroscopic clearing of popliteal cysts to avoid excessive cleaning inward and damage to the posterior blood vessels and nerves; (5)When establishing a high posterolateral approach, be careful not to damage the stop point of the lateral collateral ligament. Arthroscopy should Extending in the direction of the popliteal tendon is convenient for observing the area of the popliteal tendon hiatus, and clearing the hiatus between the popliteal tendon through the posterolateral approach, and if necessary, the curved planer is cleaned. At the same time, when entering the popliteal tendon hiatus area through the posterolateral approach, pay attention to the direction of the fibular head to prevent posterior damage to the common peroneal nerve; (6) The posterior calf abscess will form a large cavity after the removal. Bed rehabilitation training [9]; (7) Use 5\% iodophor to soak the infected particle lesions that cannot be distinguished by the naked eye in order to completely eliminate the infected lesion tissue. Limitations: This study is a retrospective analysis and a prospective randomized controlled study of arthroscopic multi-channel approach and traditional open surgery has not been carried out; the number of selected cases in this study is small, the follow-up time is limited, and its long-term efficacy still needs Follow up further. In the future, it is necessary to conduct further studies with a larger sample size to provide more evidence.

\section{Conclusion}

This study found that arthroscopic multi-channel approach combined with clearing of knee joint cavity infections and calf injection abscesses can achieve definite short-term effects, shorten the operation time, reduce the number of days in hospital, and have high patient satisfaction.

\section{Abbreviations}


TB: Tuberculosis; MTB: Mycobacterium tuberculosis; SEP: Staphylococcus epidermidis; CRP: C-reactive protein; ESR: Erythrocyte sedimentation rate; MRI: Magnetic resonance imaging; VAS: Visual analogue scale.

\section{Declarations}

\section{Ethics approval and consent to participate}

This study was approved by the Ethics Committee of The Affiliate Hospital of Southwest University \Acceptance number: KY2020243区. This study complies with the requirements and norms of the Declaration of Helsinki . Informed consent was obtained in writing from all the individual participants included in the study.

\section{Consent for publication}

Not applicable

\section{Availability of data and materials}

The datasets analyzed during the current study are available from the corresponding author on reasonable request.

\section{Competing interests}

The authors declare that they have no competing interests.

\section{Funding}

This research is supported by the Cooperation program between Sichuan University and Luzhou municipal people's government (No. 2019cdlz-17).

\section{Author contributions}

YL contributed to the data analysis and interpretation and manuscript draft. JCL and LZ contributed to the study design. Lei Lei is responsible for formulating rehabilitation training programs; PZ and FYD enrolled the patients in the study, contributed to the data collection, and participated in the interpretation of the data. The authors read and approved the final manuscript.

\section{Acknowledgements}

The authors would like to thank all the participating patient

\section{Author details}


${ }^{1}$ Department of orthopaedics, The Affiliated Hospital of Southwest Medical University, Sichuan provincial laboratory of orthopaedic Engineering, Luzhou, Si Chuan Province, People's Republic of China.

${ }^{2}$ Rehabilitation Medicine Department, The Affiliated Hospital of Southwest Medical University, Luzhou Si Chuan Province, People's Republic of China.

\section{References}

1. Gupta MN, Sturrock RD, Field M. A prospective 2-year study of 75 patients with adult-onset septic arthritis. Rheumatology (Oxford). 2001;40(1):24-30.

2. Al-Tawfiq JA, Babiker M. Incidence and bacteriologic causes of septic arthritis in a general hospital in Saudi Arabia. Ann Saudi Med. 2013;33(2):116-118.

3. Sreenivas T, Nataraj AR, Menon J. Acute hematogenous septic arthritis of the knee in adults. Eur J Orthop Surg Traumatol. 2013;23(7):803-807.

4. Sonnery-Cottet B, Archbold P, Zayni R, et al. Prevalence of septic arthritis after anterior cruciate ligament reconstruction among professional athletes. Am J Sports Med. 2011;39(11):2371-2376.

5. Sun H, Huang Z, Zhang Z, et al. A Meta-Analysis Comparing Liposomal Bupivacaine and Traditional Periarticular Injection for Pain Control after Total Knee Arthroplasty. J Knee Surg. 2019;32(3):251258.

6. Castorina S, Guglielmino C, Castrogiovanni P, et al. Clinical evidence of traditional vs fast track recovery methodologies after total arthro- plasty for osteoarthritic knee treatment.A retrospective observational study[J]. Muscles Ligaments Tendons J, 2018, 7(3): 504-513.

7. Faour M, Sultan AA, George J, et al. Arthroscopic irrigation and debridement is associated with favourable short-term outcomes vs. open management: an ACS-NSQIP database analysis. Knee Surg Sports Traumatol Arthrosc. 2019;27(10):3304-3310.

8. Bovonratwet P, Nelson SJ, Bellamkonda K, et al. Similar 30-Day Complications for Septic Knee Arthritis Treated With Arthrotomy or Arthroscopy: An American College of Surgeons National Surgical Quality Improvement Program Analysis. Arthroscopy.2018;34(1):213-219.

9. Johns B, Loewenthal M, Ho E, et al. Arthroscopic Versus Open Treatment for Acute Septic Arthritis of the Knee in Children. Pediatr Infect Dis J. 2018 May;37(5):413-418.

10. Chuang YC, Wang JL, Chen YC, et al. Characteristics and outcomes of community-onset septic arthritis in adults. J Microbiol Immunol Infect. 2009;42(3):258-264.

11. Margaretten ME, Kohlwes J, Moore D, et al. Does this adult patient have septic arthritis?. JAMA. 2007;297(13):1478-1488.

12. Taljanovic MS, Gimber LH, Omar IM, et al. Imaging of Postoperative Infection at the Knee Joint. Semin Musculoskelet Radiol. 2018;22(4):464-480.

13. Hunter JG, Gross JM, Dahl JD, et al. Risk factors for failure of a single surgical debridement in adults with acute septic arthritis. J Bone Joint Surg Am. 2015;97(7):558-564. 
14. Pool LD, Van Delft $D$, Thomassen BJ, et al.Joint needle aspiration compared to tissue samples in septic arthritis of the native knee. Acta Orthop Belg. 2020;86(1):64-68.

15. Harada K, McConnell I, DeRycke EC, et al. Native Joint Septic Arthritis: Comparison of Outcomes with Medical and Surgical Management. South Med J. 2019;112(4):238-243.

16. Johns BP, Loewenthal MR, Dewar DC. Open Compared with Arthroscopic Treatment of Acute Septic Arthritis of the Native Knee. J Bone Joint Surg Am. 2017;99(6):499-505.

17. Nair R, Schweizer ML, Singh N. Septic Arthritis and Prosthetic Joint Infections in Older Adults. Infect Dis Clin North Am. 2017;31(4):715-729.

18. Schröder JH, Krüger D, Perka C, Hufeland M. Arthroscopic Treatment for Primary Septic Arthritis of the Hip in Adults. Adv Orthop. 2016;2016:8713037.

19. Aïm F, Delambre J, Bauer T, et al. Efficacy of arthroscopic treatment for resolving infection in septic arthritis of native joints. Orthop Traumatol Surg Res. 2015;101(1):61-64.

20. Böhler C, Pock A, Waldstein W, et al. Surgical treatment of shoulder infections: a comparison between arthroscopy and arthrotomy. J Shoulder Elbow Surg. 2017;26(11):1915-1921.

\section{Tables}

Table 1 Demographic data and characteristics of patients 


\begin{tabular}{|c|c|c|c|c|c|c|}
\hline patient & Age & gender & Side & Bacterial infection & Course of disease & Follow-up, mo \\
\hline 1 & 40 & Male & Left & S. aureus & 3 & 20 \\
\hline 2 & 38 & Female & Right & S. aureus & 4 & 18 \\
\hline 3 & 50 & Male & Left & MTB & 4 & 24 \\
\hline 4 & 56 & Male & Left & E. coli & 5 & 16 \\
\hline 5 & 45 & Male & Left & S. aureus & 3 & 17 \\
\hline 6 & 39 & Male & Left & S. aureus & 7 & 18 \\
\hline 7 & 47 & Male & Right & Acinetobacter Joni & 4 & 20 \\
\hline 8 & 56 & Female & Left & S. aureus & 2 & 19 \\
\hline 9 & 33 & Male & Left & S. aureus & 5 & 12 \\
\hline 10 & 44 & Male & Left & MTB & 5 & 15 \\
\hline 11 & 55 & Male & Right & S. aureus & 5.5 & 18 \\
\hline 12 & 58 & Female & Left & SEP & 4 & 22 \\
\hline 13 & 61 & Female & Left & Oral streptococcus & 4 & 22 \\
\hline 14 & 43 & Male & Right & S. aureus & 4.5 & 20 \\
\hline 15 & 35 & Male & Left & S. aureus & 6 & 15 \\
\hline
\end{tabular}

Table 2 Comparison of ESR, CRP, VAS scores and Lysholm scores before and during the last follow-up $\square n=15 \square \pm s \square$

\begin{tabular}{|lllll|}
\hline Time & ESR $₫ \mathrm{~mm} / \mathrm{h} \rrbracket$ & $\mathrm{CRP}(\mathrm{g} / \mathrm{L})$ & VAS score & Lysholm score \\
\hline Preoperative & $44.6 \pm 10.1$ & $30.2 \pm 8.0$ & $7.0 \pm 0.9$ & $46.9 \pm 7.7$ \\
\hline Last follow-up & $11.4 \pm 3.1$ & $3.9 \pm 1.0$ & $2.1 \pm 1.2$ & $93.0 \pm 8.1$ \\
\hline Paired t test & $t=10.846$ & $t=12.040$ & $t=11.798$ & $t=-17.246$ \\
& $P<0.001$ & $P<0.001$ & $P<0.001$ & $P<0.001$ \\
\hline
\end{tabular}

\section{Figures}



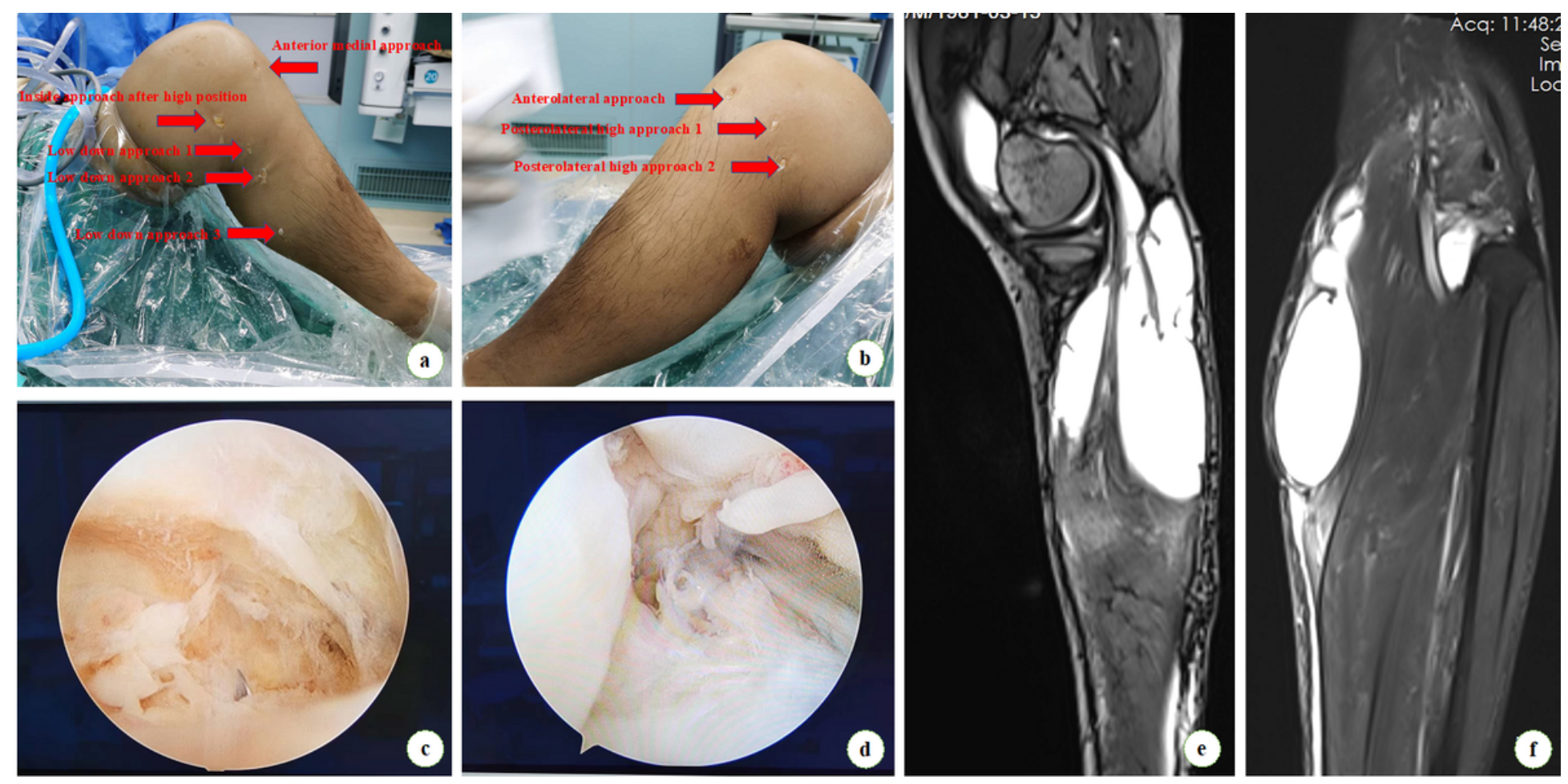

Figure 1

Patient, male, 39 years old, left knee joint suppurative arthritis with calf abscess formation $a, b$ The intraoperative medial approach and intraoperative lateral approach. c,d. Arthroscopic images. e,f. Preoperative sagittal and coronal MRI images 